\title{
FINE MOTOR RETARDATION AND DEPRESSION
}

\author{
BERNARD SABBE, ${ }^{*}$ WOUTER HULSTIJN, $\uparrow$ JACQUES VAN HOOF \\ and FRANS ZITMAN\$ \\ *Institute of Psychiatry, University of Nijmegen, Nijmegen, The Netherlands; + Nijmegen Institute for \\ Cognition and Information (NICI), University of Nijmegen, Nijmegen, The Netherlands; †Institute of Psychiatric \\ Care, Rosmalen. The Netherlands; §Institute of Psychiatry. University of Nijmegen, Nijmegen.
} The Netherlands

(Received 16 October 1995; accepted 21 March 1996)

\begin{abstract}
Summary - New computerized techniques allow the precise measurement of psychomotor retardation in patients with a major depressive episode (MDE). One such technique is the analysis of writing and drawing behaviour during figure copying tasks. In the present study, 22 inpatients with an MDE were compared to 22 normal controls. Three tasks were used: the drawing of lines and simple figures, the copying of complex figures and a task in which figures had to be rotated. Objectives were to provide support for earlier findings that the patients were slower than the controls and to explore the cognitive and motor processes involved. Two strategies were applied: analysis of the reaction time and movement time and their different components, and manipulation of the cognitive and motor demands. Patients showed considerable retardation with most of the kinematic variables. Motor deficits and cognitive slowing down contributed to this retardation. Cognitive difficulties increased with increasing complexity of the task. Copyright 0 . 1996 Elsevier Science Ltd.
\end{abstract}

\section{Introduction}

Although changes in psychomotor activity are generally viewed as important phenomena, their contribution to the diagnostic and therapeutic evaluation of patients with affective disorders remains unclear. Firstly, some authors consider psychomotor alterations to be an important symptom-on the same level as other symptoms (DSM) -or even as a core symptom of all depressive conditions or some subgroups. Secondly, a number of psychomotor symptoms have been grouped into a syndrome with its own aetiology, pathogenesis and psychoneurophysiology, comparable with fever, as an independent syndrome in different physical conditions. The "akinetic syndrome" (Bermanzohn \& Siris, 1992) and the "psychotic motor syndrome" (Günther et al., 1988) are examples of this way of thinking. This syndrome can be present in different neuropsychiatric disorders, such as Parkinson's syndrome, retarded depression and schizophrenia, but also in pharmaceutical intoxication and in association with aging. Thirdly, some investigators consider psychomotor retardation as a primary disorder in depression (Widlöcher, 1983): it can explain a large proportion of the variance in depressive symptomatology, it offers new insights into the aetiology of depression and it has a predictive value regarding the therapeutic effects of

Correspondence to: Dr Bernard Sabbe, Institute of Psychiatry, University Hospital Nijmegen, Reinier Postlaan 10. P.O. Box 9101,6500 HB Nijmegen, The Netherlands. 
antidepressive medication. These differences in opinion about the contribution of psychomotor retardation to the diagnostic process in affective disorders, are partly due to different operationalizations. The use of fairly "narrow" definitions means that only the motor behaviour manifested in facial expression, speech, gross and fine motor activity is taken into account. "Wider" definitions encompass not only motor behaviour, but also mental activities, e.g. disturbances of attention, memory and the experience of time, hedonia and vital functions, such as sleep, eating behaviour and sexual libido. In the latter definitions, the underlying assumption stresses that retardation determines both motor and mental behaviour. To define psychomotor retardation more accurately, video-analysis of the nonverbal behaviour of depressed patients and analysis of the perceptions of the clinician and their different weights in the diagnostic process (Ulrich \& Harms, 1985) offer new research possibilities.

During the past decade and since the review article by Greden \& Carroll (1981), new computerized techniques have been developed to measure psychomotor retardation (Greden, 1993). They focus on ecologically valid skills, such as speech and writing behaviour. In comparison with older methods, such as rating scales (e.g. the Salpêtrière Retardation Rating Scale), actometry and choice reaction time tasks, they allow the more precise measurement and more detailed analysis of retardation. Furthermore, they offer new scope to understand the different cognitive and motor processes involved in psychomotor retardation and to detect differences between the retardation of depressed patients and patients suffering from other neurological and psychiatric conditions. In the field of speech analysis, research is steadily increasing; several prominent features of speaking behaviour and voice sound characteristics were found to be closely related with depression and the time course of recovery (Kuny \& Stassen, 1993; Flint et al., 1993). We introduced (Van Hoof et al., 1989) an innovative technique that consists of recording and analysing writing and drawing movements, e.g. in figure copying tasks. Figure copying tasks have been used as a diagnostic tool for the assessment of psychomotor dysfunction for more than half a century. They are sensitive indicators of a wide range of neurological disorders (Lezak, 1983) and are included in a large number of tests [see Van Mier (1992) for a list of 18 tests]. Recording drawing movements during figure copying will improve our understanding of the sensory and motor processes involved in psychomotor retardation. These include a number of perceptual processes (preprocessing, feature extraction and stimulus identification), storage in the working memory, visuospatial processing and planning the next movement. These processes, which we refer to as cognitive, will take by far the largest portion of the reaction time (i.e. the time interval between the onset of stimulus presentation and the start of drawing). The other processes, denoted by the term "motor", encompass the programming, co-ordination, initiation and execution of muscle commands, as well as monitoring the visual feedback to correct errors. During movements, successive strokes are likely to be planned and programmed. Motor processes therefore consume a small part of the reaction time and most of the movement time.

In line with two earlier investigations (Van Mier \& Hulstijn, 1993; Van Hoof et al., 1993), this study compared a large group of patients with a major depressive episode to a group of normal matched controls, in an attempt to replicate earlier results and to further explore the nature of depressive retardation. Therefore, the different components of drawing 
movements were analysed and in subsequent tasks, cognitive and motor variables were manipulated independently. The study questions were: (1) Are depressed patients slower in accomplishing different drawing tasks than normal controls? (2) If so, in which aspects (kinematic variables such as the reaction time and movement time and their components) does psychomotor retardation manifest itself most prominently? (3) If the more cognitive demands and the more motor demands - in so far as they can be separated - are manipulated independently, on what points do depressed patients fail?

\section{Method}

\section{Subjects}

Forty-four subjects participated in the study: 22 patients with a major depressive episode (MDE) and 22 control subjects. At the time of the study, all the MDE-patients were hospitalized at the Clinic of Psychiatry of the University Hospital Nijmegen, The Netherlands. The diagnosis was made after an extensive and detailed auto- and heteroanamnestic interview. All the patients aged between 18 and 65 years with an MDE and a minimum score on the Hamilton Depression Rating Scale (Hamilton, 1960) of 18, who were admitted between September 1992 and April 1994, were asked for informed consent after the nature of the procedure had been fully explained. Patients were excluded if they met one of the following exclusion criteria: motor disabilities affecting writing behaviour $(N=2)$, severe cardiovascular or hepatic disease $(N=2)$, renal failure and previous unsuccessful treatment with fluoxetine $(N=4)$. Consequently, a total of eight patients were excluded; from a psychiatric point of view, they did not differ from the sample. The group that participated in the study comprised 12 male and 10 female patients. All the patients had a DSM III-R (American Psychiatric Association, 1987) diagnosis of a major depressive episode, single episode (296.2) or recurrent episode (296.3); only one patient had a bipolar disorder, depressed (296.5). The episode was severe in all the patients; 16 did not have any psychotic features (code 3), while six did have psychotic features (code 4). Two patients had a subsidiary diagnosis of previous alcohol and benzodiazepine dependence $(N=2)$. Three patients displayed a clinical state of agitation.

For each patient, there was a control subject, matched for age, sex and education.

\section{Procedure and tasks}

Once admitted to the study, all antidepressant drugs were gradually stopped, and other psychotropic drugs were slowly reduced as much as the condition of the patient allowed. Patients remained on low doses of benzodiazepines $(N=11)$, neuroleptics $(N=2)$ or a combination of the two $(N=5)$. Then, fluoxetine, at a dose of $20 \mathrm{mg}$ per day, was administered for 6 weeks. The test was performed after 1 week of fluoxetine. It consisted of a series of copying tasks with the aid of a pen on a digitizing tablet. The movement registration method is discussed in the next paragraph.

Stimuli differed in complexity and familiarity (Figure 1). Complexity was defined as the number of strokes in a figure; familiarity could be perceptual or motor: letters, for example, are well-known perceptually and motorically, while figures such as a boat or a table are 


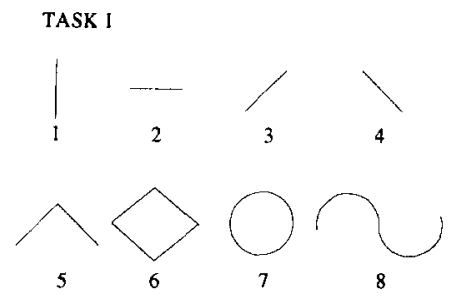

TASK II

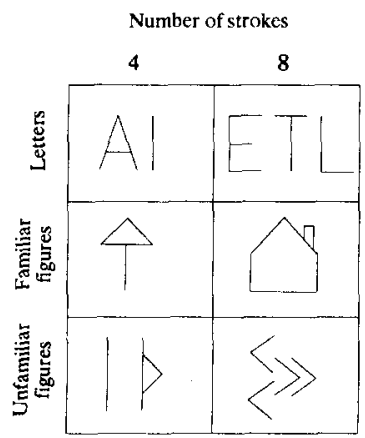

TASK III

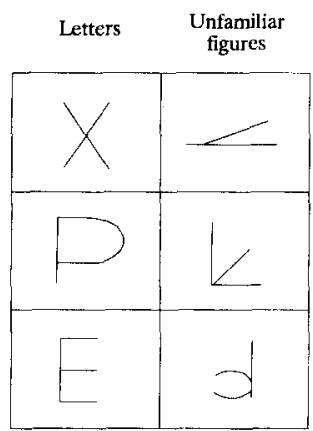

Figure 1. Stimulus designs used in Tasks I, II and III.

well-known perceptually but not motorically; novel and nonsense patterns are unknown perceptually and motorically.

Three copying tasks were assigned. Task I analysed whether retardation could be detected in the drawing of simple lines or whether more complex patterns were needed. The subjects had to copy four straight lines (one vertical, one horizontal and two diagonals) and four simple figures (Figure 1). All the stimuli were presented six times in a random order. The degree of complexity of the figures was increased in Task II to answer the second and the third questions. Three types of stimuli had to be copied: combinations of capital letters, familiar figures and novel and nonsense patterns (Figure 1). Four stimuli of each type were presented with differing complexity, two with four strokes and two with eight strokes. In Task III, a specific type of manipulation was executed that focussed on visuospatial processing. Eight figures, four combinations of letters and four figures (Figure 1) were presented, with the instruction to copy them at another angle, i.c. having rotated them through $90^{\circ}$ to the right.

In all the tasks, the subjects were instructed to draw as quickly and as accurately as possible. As soon as the pen touched the paper, the stimulus disappeared from the viewing screen. In Tasks II and III, the subject could reinspect the figure by touching the pen on a red spot at the lower right hand corner of the digitizer. He/she was asked to do this only as an "emergency" measure, if he/she felt that otherwise too many errors would be made. Prior to each task, a practice session was given in which the subject could become accustomed to 
the writing tablet and the procedure. The investigators were independent of the treatment staff. The three tasks were performed in a fixed order in $30-60 \mathrm{~min}$. They all took place between 2:00 and 5:00 p.m., to avoid any possible influences of the circadian rhythm.

\section{Recording and analysis}

The drawing movements were recorded using a Calcomp 2300 digitizer, connected to a PC (63S386) that had been specially designed to measure pen pressure, with a precision of $2 \mathrm{~g}$ (Maarse et al., 1988). The position of the pen on the digitizing tablet (axial pen force) was recorded with a frequency of $100 \mathrm{~Hz}$ and a precision of $0.2 \mathrm{~mm}$. The following movement variables were obtained: total time (TT) i.e. the sum of the reaction time and the movement time; reaction time (RT), defined as the time interval between the onset of the stimulus presentation and the moment the pen touched the paper and the pressure threshold was exceeded; and movement time (MT), i.e. the time interval between the first and last moment that the pressure threshold was exceeded. Movement time (MT) was divided into the time that the pen was moving on the paper and the pressure threshold was exceeded (MTdown), the time that the pen was on the paper but not moving (no velocity) (Pause), the time that the pen was above the paper and the pressure was below the threshold (MTup) and the video reinspection time (Video), i.e. the time that the figure was reinspected.

In Task II and Task III, the drawings were scored for errors by two independent investigators. The classification was as follows:

- A-type error: the drawing more or less resembled the actual stimulus. Distinction was made between small and severe errors:

A 1: rotation error of up to $30^{\circ}$, distortion in proportion or in relation, segmentation of parts of the stimulus, fragmentation and alignment, omissions and additions (all up to a third of the number of strokes of the original stimulus): also the drawings in which corrections had been made.

A2: rotation of more than 30 ; two-dimensionality instead of three-dimensionality; wrong letter(s); omissions, additions, reversal and distorsion in form (all up to two-thirds of the number of strokes of the original stimulus).

- B-type error: the original stimulus could not be recognized because of too many omissions or additions, or no copy had been made.

A statistical analysis was performed with two-way ANOVAs for each variable according to a repeated measurement design. The within-factors were complexity (Task I), complexity and familiarity (Task II), and familiarity (Task III), while the between-factor was group (patients versus controls).

\section{Results}

\section{Task I: lines and simple figures}

Even during the line drawing task, the mean reaction time (961 ms) and the mean movement time of the patients $(600 \mathrm{~ms})$ were significantly longer than those of the control group (758 ms and $421 \mathrm{~ms}$, respectively) (Figure 2). Table 1 presents the results of the ANOVA for total time (TT), reaction time (RT), movement time (MT), movement time 


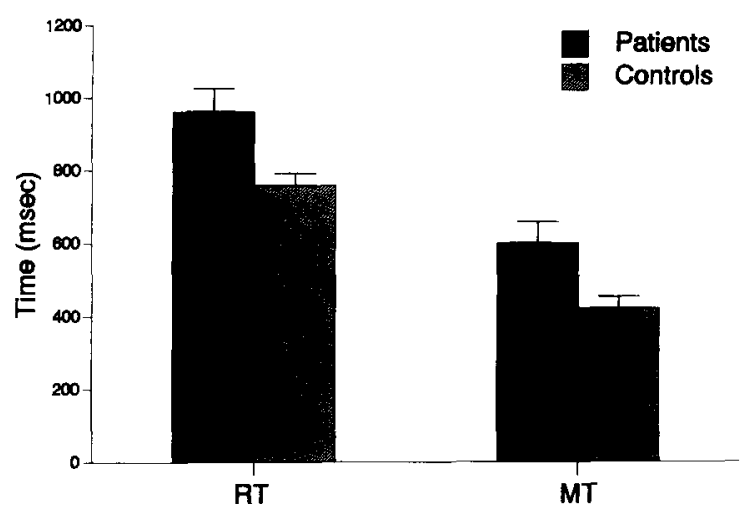

Figure 2. Mean reaction time (RT) and mean movement time (MT) of the depressed patients and their controls for copying lines in Task I.

Table 1

Analysis of Variance for the Kinematic Variables of Tasks I, II and III

\begin{tabular}{|c|c|c|c|c|c|c|c|c|}
\hline & $d f$ & TT & RT & MT & MTdown & MTup & Pause & Video \\
\hline \multicolumn{9}{|l|}{ Task I (lines) } \\
\hline Group & 1 & $10.12 * *$ & $8.09 * *$ & $7.49 * *$ & & & & \\
\hline \multicolumn{9}{|c|}{ Task I (simple figures) } \\
\hline Group (G) & 1 & $7.54^{* *}$ & $8.36^{* *}$ & $4.38 * *$ & & & & \\
\hline Complexity (C) & 1 & $100.06^{* * *}$ & $15.16^{* * *}$ & $221.63^{* * *}$ & & & & \\
\hline $\mathrm{G} \times \mathrm{C}$ & 1 & $7.73^{* *}$ & $8.27^{* *}$ & $4.59^{*}$ & & & & \\
\hline \multicolumn{9}{|c|}{ Task II (complex figures) } \\
\hline Group $(\mathrm{G})$ & 1 & $6.74^{*}$ & $4.81^{*}$ & $6.57^{*}$ & $5.12^{*}$ & 2.42 & $4.12^{*}$ & $5.37^{*}$ \\
\hline Familiarity (F) & 2 & $46.52 * * *$ & $20.27 * *$ & $24.51 * * *$ & $23.60 * * *$ & $7.12 * *$ & $12.52^{* * * *}$ & $8.33^{* *}$ \\
\hline Complexity (C) & 1 & $171.93^{* * *}$ & $53.92 * *$ & $182.79^{* *}$ & $191.98^{* * *}$ & $244.49^{* * *}$ & $28.03 * * *$ & $17.49 * * *$ \\
\hline $\mathrm{G} \times \mathrm{F}$ & 2 & 1.13 & 0.17 & 1.19 & $3.05 \dagger$ & $4.23^{*}$ & 0.75 & 1.44 \\
\hline $\mathrm{G} \times \mathrm{C}$ & 1 & $5.55^{*}$ & 2.53 & $5.35^{*}$ & $3.02 \dagger$ & 0.31 & 1.84 & $5.05^{*}$ \\
\hline $\mathrm{G} \times \mathrm{F} \times \mathrm{C}$ & 2 & $2.51 \dagger$ & 0.50 & $2.56+$ & $9.90^{* * *}$ & $3.87^{*}$ & $3.27 *$ & 1.36 \\
\hline \multicolumn{9}{|l|}{ Task III (rotation) } \\
\hline Group (G) & 1 & $5.79 *$ & $3.66^{+}$ & $4.51^{*}$ & 0.59 & $4.51 \dagger$ & 2.27 & 2.09 \\
\hline Familiarity (F) & 1 & $42.60^{* * *}$ & $7.47^{* *}$ & $5.76^{* * *}$ & $9.25^{* *}$ & $44.26^{* * *}$ & $36.77^{* * *}$ & $38.24^{* * *}$ \\
\hline $\mathrm{G} \times \mathrm{F}$ & 1 & 2.05 & 0.80 & 1.65 & 0.49 & 1.74 & $3.07 \dagger$ & 1.39 \\
\hline
\end{tabular}

Note: $F$-values for total time (TT), reaction time (RT), movement time (MT), movement time pen down (MTdown), movement time pen up (MTup), pause time (Pause) and video reinspection time (Video). $\dagger p<0.10 ;{ }^{*} p<0.05 ;{ }^{* *} p<0.01 ;{ }^{* * *} p<0.001$.

pen up (MTup), movement time pen down (MTdown), pause time (Pause) and video reinspection time (Video) for Tasks I, II and III.

The mean reaction time and the mean movement time for copying the simple figures are presented in Figure 3.

For all the figures, the depressed patients had a longer reaction time (mean RT patients: $1089 \mathrm{~ms}$, mean controls: $789 \mathrm{~ms}$ ) and a longer movement time (mean MT patients: 1559 ms, mean MT controls: $1258 \mathrm{~ms}$ ) than the control subjects. The mean effect of complexity 

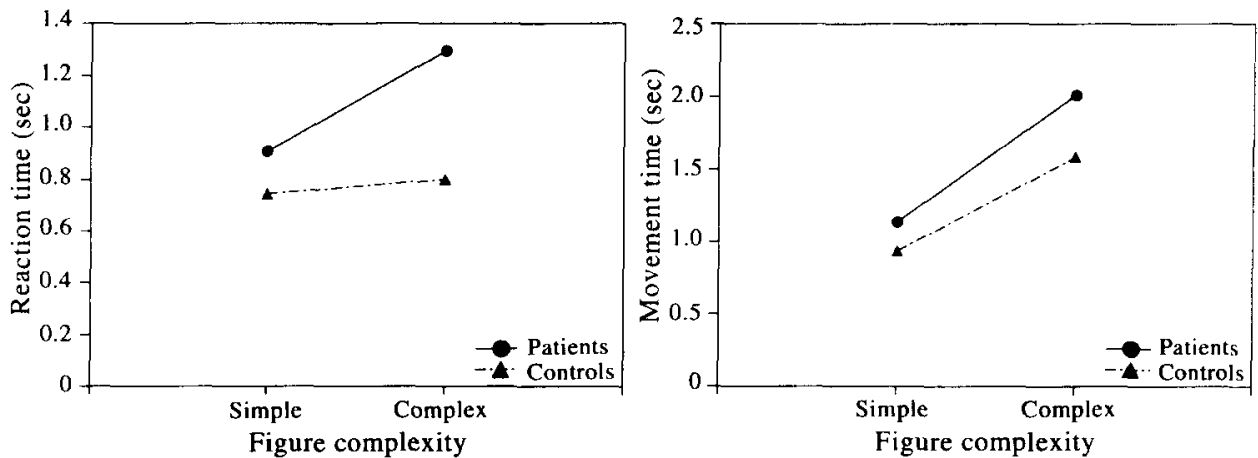

Figure 3. Mean reaction time (RT) (left panel) and mean movement time (MT) (right panel) of the depressed patients and their controls for copying simple figures in Task $\mathrm{I}$

on the RT and MT was significant $(p=0.000)$. The mean differences were larger for the more complex figures (spiral-diamond) than for the less complex figures (circle-angle), as demonstrated by the significant interactions between group and complexity (Table 1).

\section{Task II: complex stimuli}

The following variables were analysed: total time, reaction time, movement time, movement time pen down, movement time pen up, pause time and video reinspection time; also the number and severity of the errors were scored. Figure 4 presents the mean reaction time and the mean movement time of the two groups for three categories of stimuli: letter combinations, familiar figures and unknown patterns. For the other variables, the patterns were very similar.

The depressed patients and control subjects differed significantly in the reaction time (mean RT patients: $2358 \mathrm{~ms}$, mean RT: controls $1760 \mathrm{~ms}$ ), the movement time (mean MT patients: $5012 \mathrm{~ms}$, mean MT controls: $3653 \mathrm{~ms}$ ), the movement time pen down (mean MT down patients: $2938 \mathrm{~ms}$, mean MT down controls: $2228 \mathrm{~ms}$ ), the pause time (mean Pause patients: $514 \mathrm{~ms}$, mean Pause controls: $304 \mathrm{~ms}$ ) and the video reinspection time (mean
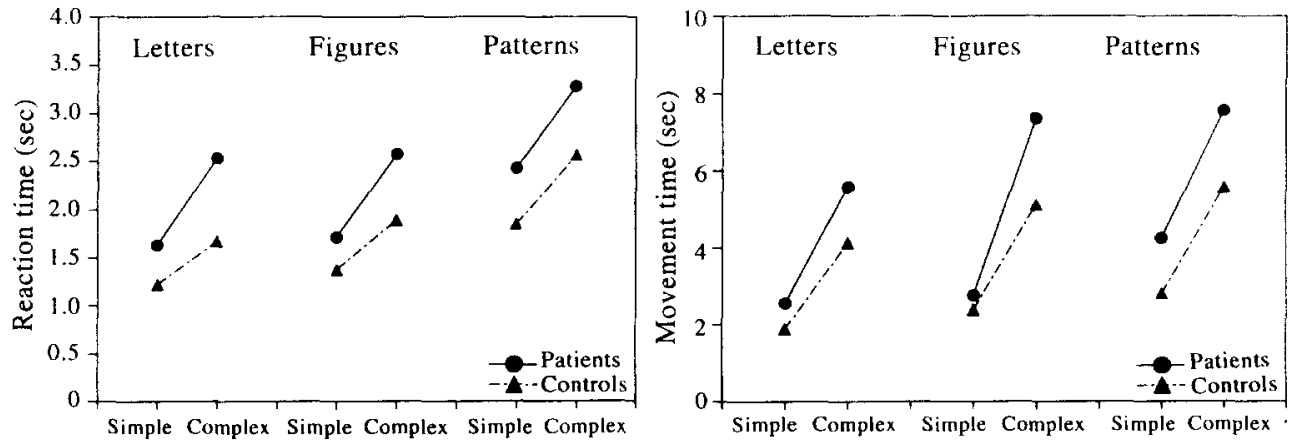

Figure 4. Mean reaction time (RT) (left panel) and mean movement time (MT) (right panel) of the patient group and control group for the three categories of stimuli of Task II: letter combinations, familiar figures and unknown patterns. 
Video patients: $647 \mathrm{~ms}$, mean Video controls: $231 \mathrm{~ms}$ ). The difference in the mean pen-up movement time (mean MTup patients: $1426 \mathrm{~ms}$, mean MTup controls: $1194 \mathrm{~ms}$ ) was not significant (Table 1). It appears that the complexity affected the MT, MTdown and the Video and that complexity and familiarity interacted with the MT, MTdown, MTup and Pause. Interaction effects between group and complexity/unfamiliarity were measured to find out whether increasing complexity/unfamiliarity had more effect on the patient group than on the control group. The following interaction effects were significant: group and complexity for MT, MTdown and Video; group and familiarity for MTup; group, familiarity and complexity for MT, MTdown, MTup and Pause. There was no difference in the number of errors or types of error between the two groups.

\section{Task III: complex stimuli; rotation}

The mean reaction time (patients: $4038 \mathrm{~ms}$, controls: $2312 \mathrm{~ms}$ ) and the mean movement time (patients: $3132 \mathrm{~ms}$, controls: $2312 \mathrm{~ms}$ ) for copying the letters and the figures after clockwise rotation through $90^{\circ}$ are presented in Figure 5.

In general, the results were similar to those for Task II: not only the reaction time and the movement time, but also the movement time pen up, discriminated between the two groups (Table 1). There were no significant interaction effects between the group and letter/figure familiarity, with the exception of a small effect for the pause time. There was no difference in the number of errors or types of error between the two groups.

\section{Clinical scales, medication and kinematic variables}

The mean score and standard deviation of the patient group on the Hamilton Depression Rating Scale was $24.4 \pm 5.8$, on the Zung Self-Rating Scale (Zung, 1965), 59.2 \pm 10.4 , and on the Salpêtrière Retardation Rating Scale $24.5 \pm 7.2$. Correlations were analysed between the results on these clinical scales, the use of neuroleptic or hypnotic medication and the values of the kinematic variables (Table 2). No correlations were found between the depression rating scales and the kinematic variables. The correlations between the Salpêtrière Retardation Rating Scale and the kinematic variables were very low. Only one correlation was found between the use of co-medication and the kinematic variables. The
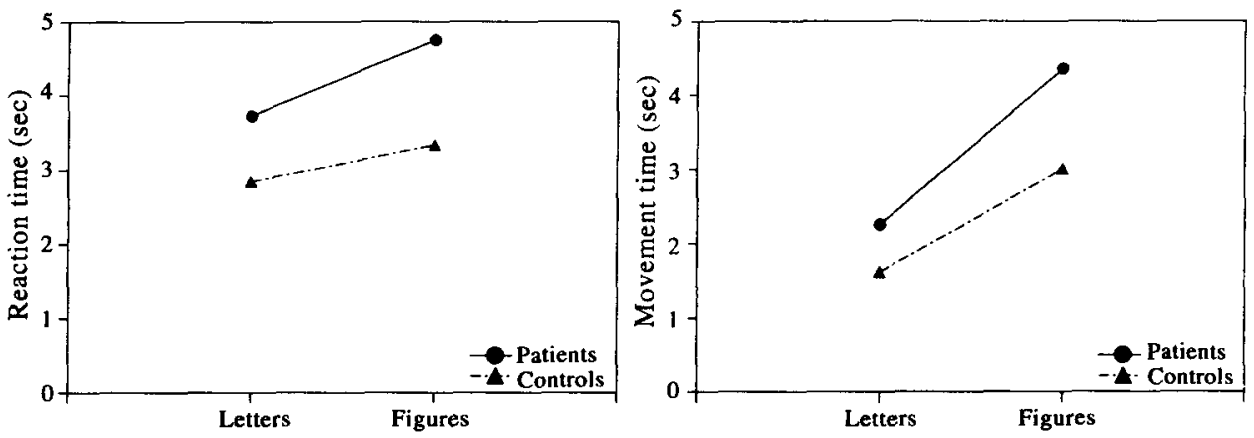

Figure 5. Mean reaction time (RT) (left panel) and mean movement time (MT) (right panel) of the patient group and control group for rotating the letters and figures of Task III. 
Table 2

Intercorrelations Between Clinical Scales, Medication and Kinematic Variables

\begin{tabular}{|c|c|c|c|c|c|c|c|c|c|c|c|}
\hline & 1 & 2 & 3 & 4 & 5 & 6 & 7 & 8 & 9 & 10 & 11 \\
\hline 1. HDRS & $-\ldots$ & 0.47 & 0.39 & -0.21 & 0.31 & -0.06 & -0.19 & -0.12 & -0.09 & 0.04 & 0.08 \\
\hline 2. Zung & & $\ldots$ & 0.30 & 0.11 & 0.34 & 0.07 & 0.35 & 0.03 & 0.21 & 0.26 & 0.33 \\
\hline 3. Salp & & & - & 0.14 & 0.07 & 0.37 & 0.29 & 0.15 & 0.23 & 0.19 & 0.13 \\
\hline 4. Neur & & & & $-\cdots$ & 0.05 & -0.06 & 0.45 & -0.16 & -0.09 & -0.08 & -0.22 \\
\hline 5. Hyp & & & & & -- & -0.01 & 0.06 & -0.08 & 0.10 & 0.14 & 0.24 \\
\hline 6. RT I & & & & & & -- & 0.61 & 0.90 & 0.81 & 0.49 & 0.56 \\
\hline 7. MT I & & & & & & & $\ldots$ & 0.57 & 0.73 & 0.46 & 0.54 \\
\hline 8. RT II & & & & & & & & $\ldots$ & 0.83 & 0.55 & 0.61 \\
\hline 9. MT II & & & & & & & & & - & 0.61 & 0.76 \\
\hline 10. RT III & & & & & & & & & & ..... & 0.46 \\
\hline 11. MT III & & & & & & & & & & & \\
\hline
\end{tabular}

Note: clinical scales: HDRS = Hamilton Depression Rating Scale; Zung = Zung Self Rating Scale; Salp = Salpêtrière Retardation Rating Scale. Use of medication: Neur = neuroleptic medication; Hyp = hypnotic medication. Kinematic variables: RT I, RT II, RT III = reaction time for Tasks I, II and III; MT I, MT II and MT III = movement time for Tasks I, II and III.

use of neuroleptics correlated significantly with the movement time for copying the simple figures. Table 2 indicates the correlations between the results on the Hamilton Depression Rating Scale, the Zung Self Rating Scale, the Salpêtrière Retardation Rating Scale, the use of neuroleptic or hypnotic co-medication and the following kinematic variables: reaction time for Tasks I, II and III and movement time for Tasks I, II and III.

\section{Discussion}

The depressed patients were much slower on most of the measures in the three drawing tasks, i.e. copying simple figures like a circle or a diamond, copying complex figures - letter combinations, familiar figures or unknown patterns - and rotating letters and figures. The depressed patients had significantly longer reaction times and movement times than the normal control subjects. Even when drawing very simple lines in different directions (horizontal, vertical and diagonal), the reaction and movement times of the patients differed significantly from those of the controls. When we considered the performance of individual patients, a wide variation and great inter-individual differences were found. Most of the patients were moderately slow, some of them were two to five times slower than the controls, and some were not retarded or were even faster than their controls. Further studies are required to determine which factors are responsible for these differences and whether these findings are also applicable to out-patients and community samples. Although it is not certain whether the medication had a positive or negative effect, we do not consider this factor to be important. After consideration of all the variables in the three tasks, only one statistically significant correlation was found between the patient subgroups without and with different classes of medication and the kinematic variables. Studies on the psychomotor and cognitive effects of fluoxetine did not demonstrate any behavioural toxicity (Hindmarch, 1995), while another study demonstrated a slight shortening of the reaction time in 
choice reaction time tasks after 1 week of treatment with this antidepressant (Kerr et al., 1993). The age range of our sample was fairly wide: $18-65$ years. There were no correlations between age and the kinematic results in the patient and control groups. In line with earlier findings, there were only weak correlations between the scores on the Salpêtrière Retardation Rating Scale and the values of the kinematic variables. This reflects the difference between the measurement of a specific short-term motor act and the rating of general verbal and non-verbal information in a questionnaire (D. Widlöcher, personal communication, 23 June 1995).

In general, these results replicate the findings in the two earlier studies (Van Mier \& Hulstijn, 1993; Van Hoof et al., 1993) and are of the same order of magnitude. This confirms the reliability of this method for studying psychomotor retardation.

Our second question, "In which aspects of the fine motor behavior does the retardation manifest itself the most prominently?", required a detailed analysis of the different kinematic variables that determine psychomotor retardation. This involves cognitive processes (perceptual, visuospatial, storage and planning) and motor processes (programming, co-ordination, initiation and execution of the motor act). The reaction time could be considered to mainly reflect cognitive processing, while the movement time was largely determined by motor processing. We found that when drawing lines and simple figures (Task I), the reaction time and movement time of the depressed patients were longer than those of the controls; this also applied to copying the more complex figures (Task II). In this test, which involved the more complex figures, the movement duration of the patients was prolonged, because their pen was moving more slowly on the paper, their pauses on the paper were longer and they needed more and longer reinspections to copy the figure. They took more time before starting to draw, took longer pauses, drew more slowly and inspected the figure more often than their controls. The resulting drawings did not differ between the two groups: the patients did not make more errors than the controls. The results were similar for the rotation task (Task III): longer reaction and movement times, especially the pen up time, i.e. the duration that the pen was held above the paper.

All these results strongly suggest that depressive retardation, apparent in all the tasks from the simpliest to the most complex, has a double origin, cognitive and motor. This motor component, (reflected by prolongation of the movement duration and specifically by the pen-down time), was less apparent in the "classical" reaction time tasks, in which the motor component often consisted of moving towards a button and pressing it. This "Pure" motor retardation was also detected in our earlier work (Van Mier \& Hulstijn, 1993; Van Hoof et al., 1993), but the pattern was slightly different. The patients did not have longer reaction times, but their movement times were prolonged because of longer pen-down times, pen-up times and video reinspection times. They also made more errors than the control group. It would seem that, when depressed patients are confronted with more complex figures, they have to choose between different strategies to cope with their difficulties. If they try to gain an overview of the whole task, their reaction will be slower and the execution of any movements will be slower, with more pauses while the pen is on the paper. Alternatively, if they start to draw without having obtained an adequate overview, the reaction time will not be prolonged, but the movements will be slower because of longer movement times on the paper or longer hesitations above the paper and longer reinspections. 
There will also be more errors in the resulting drawing. The strategy chosen will probably be influenced by factors such as the instructions, the degree of complexity of the figure and the severity of depression. In this study, the investigator gave strict instructions: he urged the participants to accomplish the tasks as quickly and as accurately as possible and to reinspect the figures only as an "emergency" measure, if no other solution was available. Also, there were fewer stimuli (12 instead of 24), and the figures were less difficult. Furthermore, the patient group that participated in this study was less depressed than those involved in previous studies. These factors can help to explain why the reaction times were prolonged: the patients put in more effort than the subjects in the past to inspect, memorize and plan the execution of the whole figure. These findings are in agreement with earlier theories about the role of motivation and sustained effort in controlled information processing (Cohen et al., 1982; Roy-Byrne et al., 1986; Hart \& Kwentus, 1987; Widlöcher \& Hardy-Bayle, 1989; Tancer et al., 1990).

In our third question, we manipulated the motor and cognitive demands of the task and detected changes in fine motor behavior. The lines in the simple task (Task I) could be considered from a cognitive- or motor point of view. Earlier results (Hulstijn et al., 1994) have suggested that only the lines that were more complex cognitively, i.e. the diagonal lines, could differentiate between patient and control groups: the patients had longer reaction times before starting to draw these lines. The actual results are analogous, but the levels of significance were only borderline. Augmenting the complexity of the figures in Task I affected the reaction time and the movement time of the depressed patients more than the controls, as was shown by the significant interactions between group and complexity. When drawing complex figures (Task II), complexity and unfamiliarity affected the movement time and specifically the pen-down time, the pen-up time and the pause time. It seems that increasing the complexity had a more disturbing influence on the movement itself, i.e. the movement time, the movement time pen down and the video reinspection time, than on the reaction time. These disturbances of the movements themselves may be related to the planning and programming processes of the next strokes, but a motor execution component may also be involved.

It can be concluded that the fine motor activities of the majority of patients with a major depressive episode, reflected in drawing tasks, were significantly retarded compared to the normal controls. Further analysis showed that retardation was already present in the very simple task of drawing a small straight line, as both reaction and movement were affected. Specific movement alterations could be isolated, and it was possible to determine which strategies the patients had chosen. These results demonstrate further evidence of the cognitive and motor burden in major depression; further research should establish the link between the cognitive and motor burden on the one hand and underlying dopaminergic and subcortical dysfunction on the other. Recording and analysing figure drawing might be a valuable addition to the methods already in use to study psychomotor retardation.

Acknowledgements-We thank Marion Pagen for helping to set up the experiment, Ans Lenders and Jacky Mullekom for testing a proportion of the subjects, and Bea Kosterman, Arjen Lelivelt, Lydia Krabbendam and Annemiek Beemsterboer for their assistance with the statistical analyses. We also thank Mrs E. van Bergen for her secretarial assistance. This study was supported in part by a grant from Eli Lilly Nederland. 


\section{References}

American Psychiatric Association (1987). Diagnostic and statistical manual of mental disorders (3rd edn, revised). Washington, DC: American Psychiatric Association.

Bermanzohn, P. C., \& Siris, S. G. (1992). Akinesia: A syndrome common to parkinsonism, retarded depression, and negative symptoms of schizophrenia. Comprehensive Psychiatry, 33, 221-232.

Cohen, R. H., Weingartner, H., Smallberg, S. A., Pickar, D., \& Murphy, D. L. (1982). Effort and cognition in depression. Archives of General Psychiatry, 33, 593-597.

Flint, A. J., Black, S. E., Campbell-Taylor, I., Gailey, G. F., \& Levinton, C. (1993). Abnormal speech articulation, psychomotor retardation, and subcortical dysfunction in major depression. Journal of Psychiatric Research, 27, $309-319$.

Greden, J. F. (1993). Psychomotor monitoring: a promise being fulfilled? Journal of Psychiatric Research, 27, 285287.

Greden, J. F., \& Carroll, B. J. (1981). Psychomotor function in affective disorders: An overview of new monitoring techniques. American Journal of Psychiatry, 138, 1441-1448.

Günther, W., Günther, R., Streck, P., Römig, H., Rödel, A. (1988) Psychomotor disturbances in psychiatric patients as a possible basis for new attemps at differential diagnosis and therapy. III. Cross validation study on depressed patients: The psychotic motor syndrome as a possible state maker for endogeneous depression. European Archives of Psychiatry and Neurological Sciences, 237, 65-73.

Hamilton, M. (1960). A rating scale for depression. Journal of Neurology, Neurosurgery and Psychiatry, 23, 5662.

Hart, R. P., \& Kwentus, J. A. (1987). Psychomotor slowing and subcortical-type dysfunction in depression. Journal of Neurology, Neurosurgery and Psychiatry, 50, 1263-1266.

Hindmarch, 1. (1995). The behavioural toxicity of the selective serotonin reuptake inhibitors. International Clinical Psychopharmacology, 9(Suppl. 4), 13-17

Hulstijn, W., Van Mier, H. I. A. J. \& Van Hoof, J. J. M. (1994). Figure copying and psychomotor retardation in depression. In C. Faure, P. Keuss, G. Lorette \& A. Vinter (Eds.), Advances in handwriting and drawing: $A$ multidisciplinary approach (pp. 477-488). Paris: Europia.

Kerr, J. S., Fairweather, D. B., \& Hindmarch, I. (1993). Effects of fluoxetine on psychomotor performance, cognitive function and sleep in depressed patients. International Clinical Psychopharmacology, 8, 341-343.

Kuny, S., \& Stassen, H. H. (1993). Speaking behavior and voice sound characteristics in depressive patients during recovery. Journal of Psychiatric Research, 27, 289-307.

Lezak, M. D. (1983). Neuropsychological Assessment (2nd edn). New York: Oxford University Press.

Maarse, F. J., Janssen, H. J. J. \& Dexel, F. (1988). A special pen for an XY-tablet. In F. J. Maarse, J. M. Mulder, W. P. B. Sjouw, \& A. E. Akkerman (Eds.), Computers in psychology: Methods, instrumentation, and psychodiagnostics (pp. 133-139). Amsterdam: Swets \& Zeitlinger.

Roy-Byrne, P. P., Weingartner, H., Bierer, L. M., Thompson, K., \& Post, R. M. (1986). Effortful and automatic cognitive processes in depression. Archives of General Psychiatry, 43, 265-267.

Tancer, M. E., Brown, T. M., Evans, D. L., Ekstrom, D., Haggerty, J. J., Pedersen, C., \& Golden, R. N. (1990). Impaired effortful cognition in depression. Psychiatry Research, 31, 161-168.

Ulrich, G., \& Harms, K. (1985). A video analysis of the non-verbal behaviour of depressed patients before and after treatment. Journal of Affective Disorders, 9, 63-67.

Van Hoof, J. J. M., Hulstijn, W., Van Mier, H. \& Pagen, M. (1989). Fine motor activity and depression. Abstracts of the IXth World Congress of Psychiatry, Athens, 2247.

Van Hoof, J. J. M., Hulstijn, W., Van Mier, J. I. A. J. \& Pagen M. (1993). Fine drawing and psychomotor retardation: Preliminary results. Journal of Affective Disorders, 23, 263-266.

Van Mier, J. I. A. J. (1992). Motor planning and movement disorders. Effects of complexity and practice in drawing tasks. PhD-thesis, K.V. Nijmegen (pp. 153-170). Nijmegen: NICI Technical Report 92-14.

Van Mier, J. I. A. J., \& Hulstijn, W. (1993). The effects of motor complexity on initiation time in writing and drawing. Acta Psychologica, 84, 231-251.

Widlöcher, D. (1983). Retardation: A basic emotional response? In J. H. Davis \& J. W. Maas (Eds.), The affective disorders (pp. 165-181). Washington, DC.: American Psychiatric Press.

Widlöcher, D., \& Hardy-Bayle, M. C. (1989). Cognition and control of action in psychopathology. European Bulletin of Cognitive Psychology, 9, 583-615.

Zung, W. K. (1965). A self-rating depression scale. Archives of General Psychiatry, 12, 63-70. 